\title{
A Novel Multi-Color Feature Selection Method for Person Re-identification
}

\author{
Yikui Zhai ${ }^{1,2}$, Lufei $\mathrm{Chen}^{1}$, Lu Cao ${ }^{1 *}$, Wengbo Deng ${ }^{1}$, Yihang $\mathrm{Zhi}^{2}$, Junying $\mathrm{Gan}^{1}$, Ying $\mathrm{Xu}^{1}$, \\ Junying Zeng ${ }^{1}$, Vincenzo Piuri ${ }^{3}$ and Fabio Scotti ${ }^{3}$ \\ ${ }^{1}$ School of Information and Engineering, Wuyi University, Guangdong, China \\ ${ }^{2}$ School of Computer, WuYi University, Jiangmen, China \\ ${ }^{3}$ Department of Information Technology Università degli Studi di Milano, Crema, Italy \\ Email: yikuizhai@163.com, lufeichen92@163.com \\ *Corresponding anthor: Caolu20001742@163.com
}

\begin{abstract}
In this paper, a novel multi-color feature selection method is proposed for person re-identification. Firstly, multi-color features, which consisting of HSV, LAB, RGB and nRnG color features, were extracted and concatenated into a whole feature vector. Then the D-optimal Partial Least Squares feature selection method was adopted to select an optimal feature subset that could minimize the variance of the regression model. Finally, an asymmetric distance model for similarity matching was utilized to observe distinctive features from a different perspective. Experimental results show that rank 1 performance of the proposed method were $48.67 \%, 63.12 \%$ and $65.04 \%$ respectively on the VIPeR, Prid_450s and CUHK01 databases, which have achieved stateof-art performances.
\end{abstract}

Keywords-Keyword: multi-color features, feature selection, D-optimal partial least squares, person re-identification

\section{INTRODUCTION}

Person re-identification (Re-ID) plays a key role in security management applications and has received increasing attention in recent years. Its goal is to identify a person (probe sample), captured by one or more cameras, while using a gallery of known candidates captured from a different camera. Pedestrian appearance can be easily obstructed by clothing, occlusion and perspective. The recognition rate is also affected by low video image resolution and light conversion, thus Re-ID still is a challenging task. Yang et al [1] proposed a novel descriptor to identify pedestrian salient color features, and had obtained good performance in addressing the problem of Re-ID. Previously, Re-ID could not sufficiently distinguish persons when they wear similarly colored cloth. Thus, a common strategy was widely applied that color and textural features should be combined to compensate each other.

Partial Least Squares is a regression technique, and is widely used for high dimensional datasets. D-Optimal Partial Least Squares are well-distributed in the important areas of the image, and it is used to select an optimal feature subset that could minimize the variance of the regression model. In this paper, a multiple feature selection method with D-optimal partial least squares is proposed for person re-identification. The proposed method takes the feature fusion and color feature model into account and makes full use of feature selection to obtain effective information for a single-shot Re-ID. The overview of the proposed person reidentification method is illustrated in Fig. 1.The rest of this paper is organized as follows. Section 2 presents the exiting person re-identification methods. Section 3 introduces the proposed multi-color features and D-optimal Partial Least Squares feature selection methods. Section 4 presents a comparison of the performances of the proposed approach with other existing methods. Section 5 concludes this paper.

\section{RELATED WORKS}

In the video surveillance environment, pedestrian appearance needs to be extracted and represented easily in a short period. Appearance-based methods assume that it was possible to simultaneously extract robust and distinctive features from different camera acquisition conditions, which is unfeasible in practice. Liao et al. [2] proposed an effective feature representation called Local Maximal Occurrence (LOMO), which could maximize the horizontal occurrence of local features to provide stable characteristics of viewpoint changes. Matsukawa et al. [3] presented a novel region descriptor, named "Gaussian of Gaussian" (GOG), to extract a set of multiple hierarchical Gaussian pixel features. Shi et al. [4] extracted the 11-dimensional color descriptor for each small block and aggregated these color descriptors into the global vector by the BoW (Big-of-Word) model. Liu et al. [5] proposed two coupled dictionaries that were related to different cameras. These were used to learn to extract robust features from different views. Karanam et al. [6] proposed a new method which was different from Liu; in which only one dictionary was specifically trained to have viewpoint invariant. Afterwards the importance of features could be computed by applying a fixed statistical model or adaptively from subsets of similar individuals and random forests. Convolutional Neural Network (CNN) has been widely used in various fields, but due to the lack of sufficient labels to train samples for person re-identification, the performance of $\mathrm{CNN}$ is not superior to traditional ones on small datasets.

Feature selection techniques consist of two methods: feature subset evaluation and individual feature ranking, the latter is more suitable to apply in person re- identification. In high-dimensional feature descriptors, the combination of multiple cues can capture subtle characteristics and benefit performance of Re-ID; however, some machine learning methods, such as the well-known Rank-SVM [7] and KISSME [8]. PLS [9] was another ideal method for dimension reduction, regression, and classification 


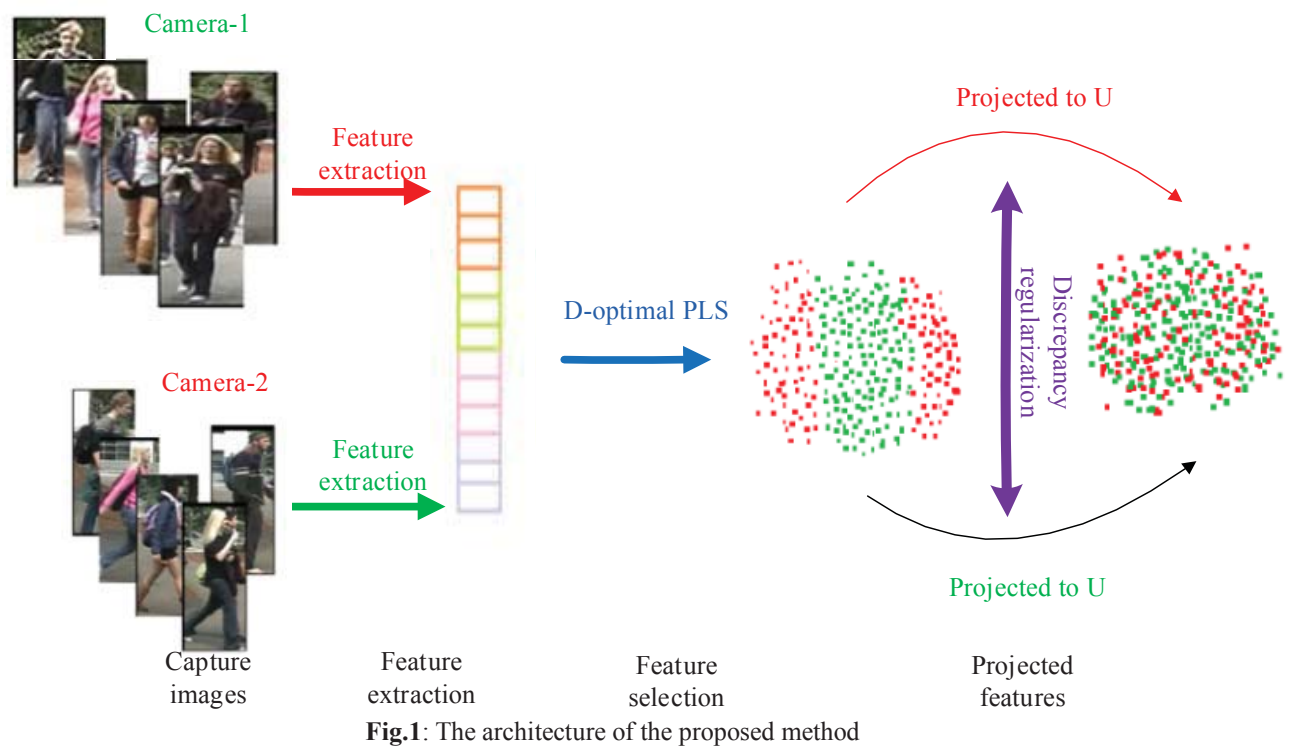

problems, and had the advantage of finding distinctive projections for intra-class information. It also had good performance in single-shot scenario. Prates and Schölkopf et al. [10] proposed Kernel PCA to nonlinearly map highdimensional feature space. Raphael et al. [11] proposed Kernel Hierarchical PCA (KHPCA) to capture high-order correlation between input variables during learning projections to a common subspace that tackles dimensional reduction. Raphael et al. [12] proposed the Cross-View Kernel feature algorithm to find a low-dimensional common space to maximize similarity of captured images.

\section{PROPOSED APPROACH}

\section{A. Multi-Color Features}

Feature representation is an important issue for person re-identification, and features from an ideal person image should be sufficiently invariant against viewing changes from different cameras. In this paper, HSV, LAB, RGB and $\mathrm{nRnG}$ color features were used to observe pedestrian images. RGB is a color benchmark in the color space. HSV and LAB are both typically used to describe color and brightness characteristics which can be generated from RGB. HSV is a more intuitive expression of the color and brightness of objects, which could better deal with image discrepancy caused by lighting issues. LAB expression range is wider than RGB, and observes all colors that is visible to the human eye. Its descriptive method is not related to perspective and equipment, so it can better handle the color discrepancy caused by different devices. RGB needs to be converted to XYZ and then converted to LAB by XYZ. $n R n G$ takes the average of the $R$ channels and $G$ channels which are effective to reduce lighting effects, defined as: $n R=R /(R+G+B), n G=G /(R+G+B)$.

A person consists of four parts: a head, an upper body, legs and feet. The upper body and legs can be uniformly divided into three layers respectively. The color information of the head and feet are insufficient. In order to capture more effective appearance information, each image is uniformly divided into seven overlapping layers. The first layer combines the head and the first layer of upper body, the last layer combines the feet and the last layer of leg. Other overlapping layers systematically combine the bottom part of upper layer and highest part of next layer. Each layer extracts HSV, LAB, RGB and $\mathrm{nRnG}$ color information, then different color features merge in each layer separately. Finally those color characteristics are cascaded into a whole multi-color feature vector.

\section{B. D-Optimal Partial Least Squares}

\section{1) Partial Least Squares}

PLS is a method that can both extract features and regression, which is used for high-dimensional data whose number of samples is far fewer than the number of features [13]. The linear PLS model can be expressed as:

$$
\begin{aligned}
& X=T P^{T}+E \\
& Y=U Q^{T}+F
\end{aligned}
$$

where $X\left(X \in R^{n \times p}\right)$ is the zero-mean feature matrix, $Y\left(Y \in R^{n \times q}\right)$ is response matrix, and matrices $T$ and $U$ contain computed score vectors of $X$ and $Y$ that are projections of $n$ samples in $d$-dimensional orthogonal subspace. $P$ and $Q \in R^{m \times f}$ are $X$ and $Y$ loadings, and $E$ and $F \in R^{n \times m}$ are the residuals of $X$ and $Y$. The inner relation between $T$ and $U$ is a linear regression model, $T$ is called predictor of $U$. If $B$ is the regression coefficient of inner relationship between scores, then $U$ can be written as:

$$
U=T B
$$

Taking (3) into (2), we get:

$$
Y=T B Q^{T}+F=T \widehat{B}+F
$$

where $\widehat{B}=B Q^{T}$. The least squares estimate of $\widehat{B}$ is given by:

$$
\widehat{B}=\left(T^{T} T\right)^{-1} T^{T} Y
$$

$T$ is obtained by a linear combination of the variables in $X$ with the weights $W^{\prime}$ as shown in n (6).

$$
T=X W^{\prime}
$$




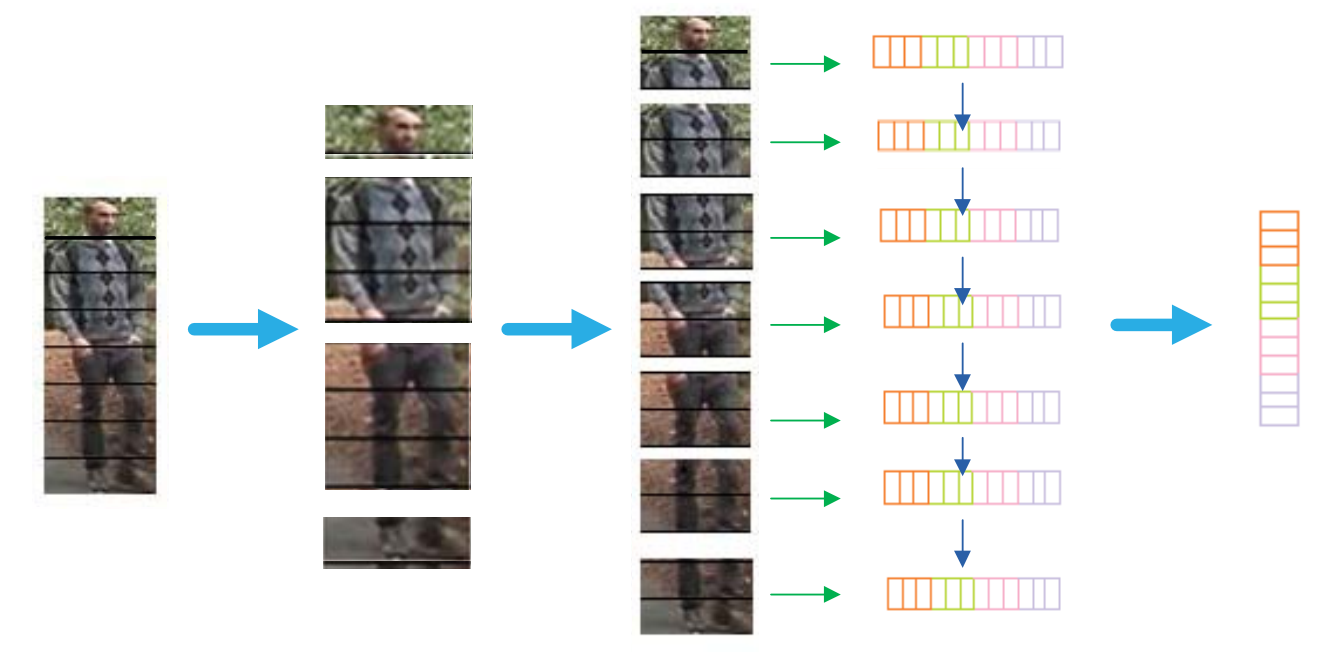

original image

segmentation

RGB HSV LAB nRnG

concatenated feature

Fig. 2 The process of multi-color feature extraction

NIPALS [14] and SIMPLS [15] are the two most commonly used algorithms to obtain the PLS model, and the relationship between two is given by (6), in which $W^{\prime}$ is as follows:

$$
W^{\prime}=W\left(P^{T} W\right)^{-1}
$$

As a result, the linear regression of PLS can be expressed as:

$$
\widehat{Y}=T \widehat{B}=T\left(T^{T} T\right)^{-1} T^{T} Y
$$

\section{2) D-Optimal PLS}

Let $\tau$ represent a feature indices subset of the set $\{1,2,3, \cdots, p\}$ containing exactly $k$ elements. The matrix $X_{\tau}$ of feature subset is expressed as:

$$
X_{\tau}=X_{n \times p} \Gamma_{p \times k}
$$

where $\Gamma$ is a selected $k$-column matrix from $p$ features, which contains a single entry in a row indexed by one element in $\tau$ and zero elsewhere.

Consider a linear Partial Least Squares regression model:

$$
Y=X \alpha+\eta
$$

where $Y$ is the response matrix, $X$ is the feature matrix, $\alpha$ is regression coefficient vector, and $\eta$ is noise vector containing mean zero and covariance of $\delta^{2} I_{n}$. The estimation of PLS regression coefficients is obtained by substituting for $T_{\tau}$ from (7) into (5).

$$
\widehat{\alpha}_{\tau}=\Gamma W_{\tau}^{\prime}\left(T_{\tau}^{T} T_{\tau}\right)^{-1} T_{\tau}^{T} Y=\Gamma W_{\tau \tau}^{\prime} T_{\tau}^{T} Y
$$

By substituting for $Y$ from (10) in (11), the mean of PLS estimate is given by:

$$
E\left[\widehat{\alpha}_{\tau}\right]=\Gamma W_{\tau}^{\prime} T_{\tau}^{T} X \alpha+\Gamma W_{\tau}^{\prime} T_{\tau}^{T} E[\eta]=\Gamma W_{\tau}^{\prime} T_{\tau}^{T} X \alpha
$$

where $\Gamma W_{\tau}^{\prime} T_{\tau}^{T} X \alpha$ and $\eta$ are assumed negligibly correlated. Thus the covariance of $\hat{\alpha}_{\tau}$ is given by:

$$
\begin{aligned}
\operatorname{cov}\left(\hat{\alpha}_{\tau}\right) & =E\left[\left(\hat{\alpha}_{\tau}-E\left[\hat{\alpha}_{\tau}\right]\right)\left(\hat{\alpha}_{\tau}-E\left[\hat{\alpha}_{\tau}\right]\right)^{T}\right] \\
& =E\left[\hat{\alpha}_{\tau} \hat{\alpha}_{\tau}^{T}\right]-E\left[\hat{\alpha}_{\tau}\right] E\left[\hat{\alpha}_{\tau}^{T}\right] \\
& =\delta^{2} \Gamma W_{\tau}^{\prime} W_{\tau}^{T T} \Gamma^{T}
\end{aligned}
$$

For a new sample $(x, y), y=x^{T} \alpha+e$ and $\hat{y}=x^{T} \hat{\alpha}_{\tau}$, the bias, variance, and noise components are the decomposition of mean squared prediction error of PLS respectively.

$$
\begin{aligned}
E\left[(y-\hat{y})^{2}\right] & =x^{T} E\left[\left(\alpha-\hat{\alpha}_{\tau}\right)\left(\alpha-\hat{\alpha}_{\tau}\right)^{T}\right] x+\delta^{2} \\
& =\operatorname{Bias}^{2}+x^{T}\left(\delta^{2} \Gamma W_{\tau}^{\prime} W_{\tau}^{\prime T} \Gamma^{T}\right) x+\delta^{2}
\end{aligned}
$$

where

$$
\operatorname{Bias}^{2}=x^{T}\left(I_{p}-\Gamma W_{\tau}^{\prime} T_{\tau}^{T} X\right) \alpha \alpha^{T}\left(I_{p}-X^{T} T_{\tau} W_{\tau}^{\prime T} \Gamma^{T}\right) x
$$

Since the squared prediction error is directly proportional to $\operatorname{cov}\left(\hat{\alpha}_{\tau}\right)$, the covariance of PLS regression coefficients can be minimized to minimize the prediction error. The minimization of the model variance can help high dimensional features to avoid overfitting. Through various criterions, the Optimal Experiment Design proposes that this covariance minimization can be obtained by optimizing the eigenvalues of $\Gamma W_{\tau}^{\prime} W_{\tau}^{\prime T} \Gamma^{T}$.

Substituting $W_{\tau}^{\prime}$ into equation (6), we can get:

$$
\begin{aligned}
\operatorname{eigval}\left(W_{\tau}^{\prime} W_{\tau}^{\prime T}\right) & =\operatorname{eigval}\left[W_{\tau}\left(P_{\tau}^{T} W_{\tau}\right)^{-1}\left(\left(P_{\tau}^{T} W_{\tau}\right)^{-1}\right)^{T} W_{\tau}^{T}\right] \\
& =\operatorname{eigval}\left[W_{\tau} W_{\tau}^{T}\left(P_{\tau} P_{\tau}^{T}\right)^{\dagger} W_{\tau} W_{\tau}^{T}\right] \\
& =\operatorname{eigval}\left[\left(P_{\tau} P_{\tau}^{T}\right)^{\dagger}\right]
\end{aligned}
$$

While eigval () is the eigenvalues of a matrix, the matrices $W_{\tau}^{\prime} W_{\tau}^{\prime T}$ and $\left(P_{\tau} P_{\tau}^{T}\right)^{\dagger}$ both have the same non-zero eigenvalues. The symbol $\uparrow$ represents the Moore-Penrose inverse. The D-optimality of PLS is given by:

$$
\underset{\Gamma}{\arg \max } \operatorname{det}^{\dagger}\left[\left(P_{\tau} P_{\tau}^{T}\right)^{\dagger}\right]
$$

Where $\operatorname{det}^{\dagger}()$ is pseudo-determinant, obtained by multiplying the non-zero eigenvalues of the matrix.

After obtaining multi-color features from HSV, LAB, RGB and $n R n G$ color space, D-optimal Partial Least Squares are applied for selecting the optimal feature subset that could minimize the variance of the regression model.

\section{EXPERIMENTS AND ANALYSIS}

\section{A. Datasets and Evaluation Protocol}

VIPeR dataset [16]. VIPeR dataset contains 632 pairs of pedestrians, captured by two cameras in an outdoor 


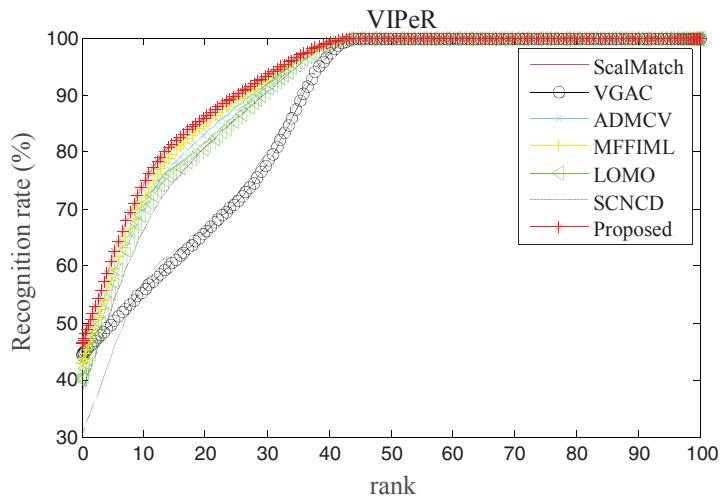

Fig. 3. Performance comparison of proposed

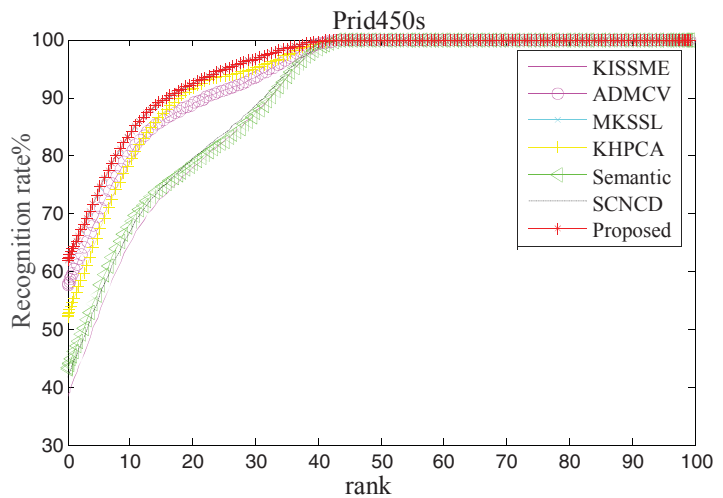

Fig. 4. Performance comparison of proposed and other methods on Prid 450s database and other methods on VIPeR database

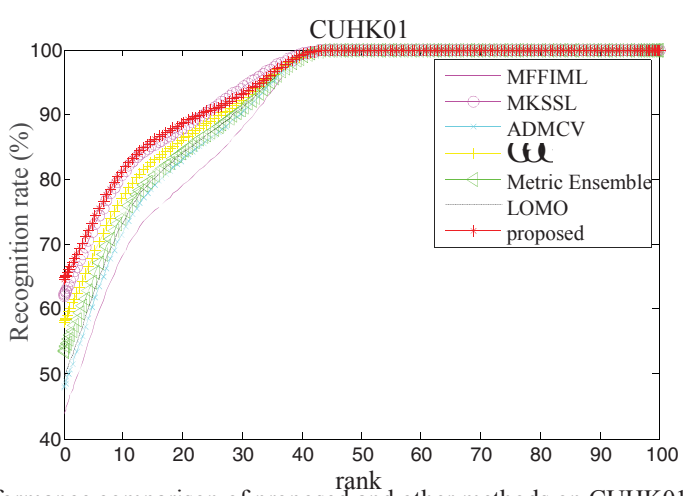

Fig. 5. Performance comparison of proposed and other methods on CUHK01 database

environment, and all images are scaled to $128 \times 48$. The VIPeR database is one of the most challenging databases in person re-identification because it includes a variety of challenges such as lighting, perspective and posture, and is usually used in supervised and unsupervised person reidentification.

Prid_450s database [17]. Prid_450s database is also available outdoors by two cameras as VIPeR, but its images are more realistic. It contains 450 pairs of pedestrians, each image taken from different cameras. CUHK01 database [18]. CUHK01 database was taken by two cameras in the campus, each pedestrian has two images under each camera. It contains 971 pairs of pedestrians, and the size of each image is $160 \times 60$. Experimental Setup. Similar to other works in recent literature, the data were tested an average of 10 times at random under the same size of training and testing subsets to obtain more stable results. Considering reidentification as a ranking problem, the performance is measured in Cumulative Matching Characteristic (CMC) curves. Since all databases evaluation protocols are consistent with ADMCV [19] in the experiments, half of the people are randomly selected every time to form a training set, leaving half of them to form a test set. The experiment used a single-shot to evaluate, meaning each pedestrian only has one image under each camera. The dimensionality of whole feature vector was 27622 and the dimensionality of selecting was set as $d=100$.

\section{B. Experimental Results and Analysis}

We will first compare the proposed method with
ADMCV [19], including add and not add feature selection method on VIPeR, Prid_450s and CUHK01 datasets respectively. Its rank 1 performance is shown in table 1. It's clear that the proposed method without D-PLS is better than ADMCV. Also ADMCV plus D-PLS is actually worse than proposed method. The result is that the method of multicolor features can extract more effective appearance information, and D-optimal Partial Least Squares feature selection can improve rank recognition rate.

TABLE 1. The RANK 1 Result of Proposed Method With ADMCV ON THREE OPEN DATASETS

\begin{tabular}{llll}
\hline \multicolumn{1}{c}{ Method } & \multicolumn{1}{c}{ VIPeR } & Prid_450s & CUHK01 \\
\hline ADMCV & 43.29 & 57.60 & 47.80 \\
Proposed without D-PLS & 43.96 & 58.04 & 54.69 \\
ADMCV+D-PLS & 45.45 & 60.63 & 62.88 \\
Proposed & 48.67 & 63.12 & 65.04 \\
\hline
\end{tabular}

Having compared the proposed method with ADMCV [19], LOMO [2], MFFIML [20], SCNCD [1], VGAC [21], and ScalMatch [22] respectively on VIPeR dataset, it is evident from Table 2 and Fig. 3 that the proposed D-optimal PLS always surpasses the aforementioned methods with a clear margin in the Re-ID performance. ADMCV [19] exacted RGB, HSV, YCbCr, LAB and YIQ five color features and 16 Gabor texture filters from each image which was then divided into 18 layers; VGAC [21] was based on the characteristics of the middle layer to cite the vertical global apparent constraints, and the characteristics that vertical appearance information from different images from 
the same person was similar to solve as an intra-class problem. The proposed method could extract more color characteristics information, and had higher recognition rate, and D-optimal PLS was adopted to reduce dimensionality and select a more effective feature set. This suggests that our D-optimal PLS self-adaptive modeling is superior to conventional adaptive for Re-ID.

TABLE 2. TOP RANKED APPROACHES ON VIPER

\begin{tabular}{lllll}
\hline \multicolumn{1}{c}{ Algorithm } & \multicolumn{1}{c}{ Rank1 } & \multicolumn{1}{c}{ Rank5 } & Rank10 & Rank20 \\
\hline ScalMatch[22] & 30.28 & - & 65.54 & 79.15 \\
SCNCD[1] & 37.81 & 68.55 & 81.28 & 90.43 \\
LOMO [2] & 40.04 & - & 80.51 & 91.08 \\
MFFIML[20] & 42.70 & 74.53 & 85.42 & 92.83 \\
ADMCV [19] & 43.29 & 72.66 & 83.51 & 92.18 \\
VGAC[21] & 44.38 & 55.80 & 65.89 & 76.39 \\
Proposed & 48.67 & 77.98 & 86.49 & 93.54 \\
\hline
\end{tabular}

We evaluated the generality of D-optimal PLS instantiated by integrating different learning methods on Prid_450s dataset. Six popular state-of-the-art methods were considered: ADMCV [19], Semantic [4], SCNCD [1], KHPCA [11], MKSSL [23] and KISSME [8]. It is clearly illustrated in Fig.4 that our proposed method, which incorporated different color features, achieved better Re-ID result than other methods. The Re-ID results are reported in Table 3 showing that the rank 1 of the proposed method is $63.12 \%$, which is nearly $6 \%$ higher than ADMCV [19]. Also the recognition rates of rank 1 to rank 20 are significantly higher than other methods. Experimental results show that compared to other methods, the proposed method has certain advantages and flexibility in the feature extraction model.

TABLE 3. TOP RANKED APPROACHES ON PRID_450S

\begin{tabular}{lllll}
\hline \multicolumn{1}{c}{ Algorithm } & Rank1 & Rank5 & Rank10 & Rank20 \\
\hline KISSME[8] & 38.49 & 67.20 & 78.09 & 86.89 \\
SCNCD[1] & 41.61 & 68.92 & 79.48 & 87.85 \\
Semantic[4] & 43.17 & 70.52 & 78.28 & 86.22 \\
KHPCA [11] & 52.23 & 80.96 & 92.80 & 94.48 \\
ADMCV [19] & 57.60 & 82.67 & 89.24 & 93.20 \\
MKSSL [23] & 61.62 & 85.71 & 92.60 & 96.71 \\
Proposed & 63.12 & 85.82 & 92.62 & 96.71 \\
\hline
\end{tabular}

The proposed method was compared with ADMCV [19], MFFIML [20], Metric Ensemble [24], GOG [11], LOMO [2] and MKSSL [23] respectively in CUHK01 dataset, the result is illustrated in Fig 5 that the recognition rate of the proposed method was significantly higher than state-of-the-art methods. Table 4 shows that the rank 1 of the proposed method is $65.04 \%$, and the value of rank 1 to rank 5 is higher than that of ADMCV [19] and other state-of-theart methods. It is shown that the proposed method attribute this improvement to the better representation from multiple feature fusion and selection, which proves that the experimental method had a relatively good recognition effect.
TABLE 4. TOP RANKED APPROACHES ON CUHK01

\begin{tabular}{lllll}
\hline \multicolumn{1}{c}{ Algorithm } & Rank1 & Rank5 & Rank10 & Rank20 \\
\hline MFFIML[20] & 43.71 & 70.85 & 79.02 & 87.39 \\
ADMCV[19] & 47.80 & 74.16 & 83.44 & 89.92 \\
LOMO [2] & 49.25 & 75.74 & 84.26 & 90.81 \\
Metric Ensemble[24] & 53.41 & 76.42 & 84.46 & 90.50 \\
GOG[11] & 57.80 & 79.12 & 86.23 & 92.17 \\
MKSSL[23] & 62.05 & 82.93 & - & 94.28 \\
Proposed & 65.04 & 83.91 & 89.64 & 93.87 \\
\hline
\end{tabular}

\section{CONCLUSION}

In this work, we tackled the Re-ID problem using multicolor features with the D-optimal partial least squares feature selection method. To the best of our knowledge, this is the first time that D-optimal partial least squares has been employed to solve the Re-ID problem. After concatenating HSV, LAB, RGB and $\mathrm{nRnG}$ color space, D-optimal partial least squares were utilized to select an optimal feature subset. Comparing with the state-of-the-art methods, the experimental results verify the superiority and advantages of our proposed method based on three challenging person reidentification benchmarks.

\section{ACKNOWLEDGMENT}

This work is supported by National of Nature Science Foundation Grant (No.61372193, No.61771347), Youth Innovation Talent Project of Guangdong Province (No.2016KQNCX171).

\section{REFERENCES}

[1] Y. Yang, J. Yang, J. Yan, S. Liao, D. Yi, S. Z. Li, "Salient Color Names for Person Re-identification," Proc. European Conference on Computer Vision, Vol. 8689, No.9. Springer, Cham. 2014, pp. 536551.

[2] S. C. Liao, Y. Hu, X. Y. Zhu, S. Z Li., "Person re-identification by Local Maximal Occurrence representation and metric learning," Pro. 2015 IEEE Conference on Computer Vision and Pattern Recognition, Boston, MA, USA, Vol. 8, No.4, 2015, pp.2197-2206.

[3] T. Matsukawa, T. Okabe, E. Suzuki, Y. Sato, "Hierarchical Gaussian Descriptor for Person Re-identification," Proc. 2016 IEEE Conference on Computer Vision and Pattern Recognition, Las Vegas, NV, USA, 2016, pp.1363-1372.

[4] Z. Y. Shi, T. M. Hospedales, T. Xiang, "Transferring a semantic representation for person re-identification and search," Proc. IEEE Conference on Computer Vision and Pattern Recognition, Boston, MA, USA, Vol.07, No.12, 2015, pp.4184-4193.

[5] X. Liu, M. Song, D. Tao, X. Zhou, C., Chwn, J Bu, "Semisupervised coupled dictionary learning for person re-identification," Proc. IEEE Conference on Computer Vision and Pattern Recognition, Columbus, OH, USA, 2014, pp.3550-3557.

[6] S. Karanarm, Y. Li, R. J. Radke, "Person re-identification with discriminatively trained viewpoint invariant dictionaries," Proc. IEEE International Conference on Computer Vision, Boston, MA, USA, 2015, pp.4516-4524. 
[7] B. Prosser, W. S. Zheng, S. G. Gong, T. Xiang, Q Mary, "Person reidentification by support vector ranking," Proc. the British Machine Vision Conference. Vol. 42, No.7, 2010, pp.1-11.

[8] M. Köstinger, M. Hirzer, P. Wohlhart, P. M. Roth, H. Bischof, "Large scale metric learning from equivalence constraints," Proc. 2012 IEEE Conference on Computer Vision and Pattern Recognition, Providence, RI, USA, 2012, pp.22882295.

[9] K. P. Bennett, M. J. Embrechts, “An optimization perspective on kernel partial least squares regression," Advances in Learning Theory: Methods, Models and Applications, Vol. 3, No.10, 2003, pp. 227-250.

[10] B. Schölkopf, A. Smola, K. R. Muller, "Nonlinear component analysis as a kernel eigenvalue problem," Neural Computation, Vol. 10, No.5, 1299-1319. 1998

[11] R. F. Prates, W. R. Schwartz, "Kernel Hierarchical PCA for Person Re-identification," Proc. International Conference on Pattern Recognition. 2017, pp. 1-8.

[12] R. F. Prates, W. R. Schwartz, "Kernel Partial Least Squares for Person Re-identification," Proc. 13th IEEE International Conference on Advanced Video and Signal Based Surveillance, Colorado Springs, CO, USA, 2016, pp.249-255.

[13] V. K. Nagarajia, W. Abd-Almageed, "Feature Selection using Prtaial Least Squares Regression and Optimal Experiment Design," Proc. International Joint Coference on Neural Networks, Killarney, Ireland, 2015, pp.1-8.

[14] S. Wold, "PLS-regression: a basic tool of chemometrics," Chemome-trics and Intelligent Laboratory Systems, Vol. 58, No.2, 2001, pp.109-130.

[15] D. S. Jong, "SIMPLS: An alternative approach to partial least squares regression," Chemometrics and Intelligent Laboratory Systems, Vol. 18, No.3, 1993, pp.251-263.

[16] D. Gray, S. Brennan, H. Tao, "Evaluating appearance models for recognition, reacquisition, and tracking," Proc. 10th International Workshop on Performance Evaluation for Tracking and Surveillance, Vol.3, 2007, pp.41-47.
[17] P. M. Roth, M. Hirzer, M. Koestinger, C. Beleznai, H. Bischof, "Mahalanobis distance learning for person re-identification," Proc. IEEE Conference on Computer Vision and Pattern Recognition, Columbus, OH, USA, 2014, pp.247-267.

[18] W. Li, R. Zhao, X. Wang, "Human Re-identification with Transferred Metric Learning," Proc. Asian Conference on Computer Vision, LNCS Vol. 7724. Springer, Berlin, Heidelberg, 2013, pp.31-44.

[19] Y. C. Chen, W. S. Zheng, J. H. Lai, P. Yuen, “An Asymmetric Distance Model for Cross-view Feature Mapping in Person Reidentification," IEEE Transactions on Circuits and Systems for Video Technology, 2016, pp.1-12.

[20] M. B. Qi, L.F. Hu, J. G. Jiang, "Person re-identification based on multi-features fusion and independent metric learning," Journal of Image and Graphics, Vol. 21, No.11, 2016, pp.1464-1472.

[21] C. X. Fan, Y.J. Chen, L. Cao, Y.L. Miu, "Person re-identification based on visual perceptual model," Computer Engineering and Applications, Vol. 52, No.6, 2016, pp.156-161.

[22] R. Zhao, W. L Ouyang, X. G. Wang, "Person Re-identification by Salience Matching," Proc. IEEE International Conference on Computer Vision, Sydney, NSW, Australia, 2013, pp.2528-2535.

[23] X. Yang, M. Wang, R. C. Hong, "Enhancing Person Reidentification in a Self-trained Subspace," ACM Transactions on Multimedia Computing, Communications, and Applications. Vol.12, No.3, 2017, pp.1-11.

[24] S. Paisitkriangkrai, C. Shen, A. V. D. Hengel, "Learning to rank in person re-identification with metric ensembles," Proc. 2015 IEEE Conference on Computer Vision and Pattern Recognition, Boston, MA, USA, 2015, pp.1846-1855. 